\title{
DYNAMIC SMART GRID COMMUNICATION PARAMETERS BASED COGNITIVE RADIO NETWORK
}

\author{
Haider Tarish Haider ${ }^{a, *}$, DhiaA Halboot Muhsen $^{a}$, \\ Haider Ismael Shahadi ${ }^{b}$, Ong Hang $\mathrm{SeE}^{c}$, Wilfried Elmenreich ${ }^{d}$ \\ ${ }^{a}$ University of Mustansiriyah, Department of Computer Engineering, 10001 Baghdad, Iraq \\ ${ }^{b}$ University of Karbala, Department of Electrical and Electronic Engineering, 56001 Karbala, Iraq \\ ${ }^{c}$ Universiti Tenaga Nasional, Department of Electronics and Communication Engineering, 43000 Kajang, \\ Selangor, Malaysia \\ ${ }^{d}$ Alpen-Adria-Universität Klagenfurt, Institute of Networked $\&$ Embedded Systems/Lakeside Labs, 9020 \\ Klagenfurt, Austria \\ * corresponding author: haiderth@uomustansiriyah.edu.iq
}

\begin{abstract}
The demand for more spectrums in a smart grid communication network is a significant challenge in originally scarce spectrum resources. Cognitive radio (CR) is a powerful technique for solving the spectrum scarcity problem by adapting the transmission parameters according to predefined objectives in an active wireless communication network. This paper presents a cognitive radio decision engine that dynamically selects optimal radio transmission parameters for wireless home area networks (HAN) of smart grid applications via the multi-objective differential evolution (MODE) optimization method. The proposed system helps to drive optimal communication parameters to realize power saving, maximum throughput and minimum bit error rate communication modes. A differential evolution algorithm is used to select the optimal transmission parameters for given communication modes based on a fitness function that combines multiple objectives based on appropriate weights. Simulation results highlight the superiority of the proposed system in terms of accuracy and convergence as compared with other evolution algorithms (genetic optimization, particle swarm optimization, and ant colony optimization) for different communication modes (power saving mode, high throughput mode, emergency communication mode, and balanced mode).
\end{abstract}

KEYWORDS: Smart grid, home area network, cognitive radio, decision engine, differential evolution.

\section{INTRODUCTION}

The integration of information and communication technology (ICT) systems has transformed the traditional power grid into a smart grid [1. ICT systems enable the efficient use of energy by deploying intelligent devices and control systems to automate power grids for energy and cost saving [2]. Furthermore, advanced communication systems contribute to the interaction between utility companies and customers. Consequently, customers can save energy and cost, while utility companies can maintain system reliability and resilience. Wireless technologies are a preferred option in several parts of a smart grid to provide flexible and low-cost data communication and networking [3]. In a smart grid, three main wireless communication networks exist, ranging from those used in a home area network (HAN) to connect various appliances and devices within a home 4, to a neighbourhood area network (NAN), directly connecting multiple end users (HANs) in specific areas to the data concentrator/substation and, ultimately to the wide area network (WAN), which connects many NANs to the central control unit.

Due to the rapid development of smart grids, an increasing number of smart meters have been installed in the HAN, thus the amount of data to be transmitted is growing rapidly. Furthermore, the emerging new paradigms, such as the internet of things (IoT), device-to-device (D2D) communication, and smart appliances, are expected to have a massive spectrum demand [5]. Therefore, more spectrum bands are required to provide an accurate and flexible wireless communication in the smart grid, and this requirement presents a significant challenge in originally scarce spectrum resources [6. Cognitive radio (CR) has provided a powerful technique to overcome the stringent spectrum resource [7, 8. The CR has the possibility to sense the wireless environment parameters and adapt intelligently for providing an optimized service that improves the communication performance [9]. Spectrum sensing and spectrum decision operations involve the cognitive cycle; its applications are not limited to licensed bands and can be applied to cognitive radio users while accessing unlicensed bands to increase the efficiency and capacity [10].

The CR-based communication in smart grids has been investigated in terms of various aspects, such as architecture management [11, 12, channel selection 13, reliability of event estimation [14, 15, security and protection [16, 17], and multimedia commu- 
nications [18. However, the optimal selection of the HAN network parameters based on the communication environment has not been thoroughly investigated despite it being one of the most important requirements of smart grid communications.

There are many works for a cognitive radio application in a smart grid. In 19, an approach based on a cellular learning automata for designing cognitive engines in the cognitive peer-to-peer networks is proposed. In [20, a dynamic channel selection algorithm for the CR-based smart grid communication network is proposed. The proposed algorithm is based on a fuzzy inference system to select suitable channel parameters including bandwidth, SNR and probability of missed detection. In [14, a reliable spectrum access and reaching consensus with the cognitive radio sensor and actor nodes are discussed. Furthermore, a consensus scheme is proposed to increase the reliability by enabling a consensus convergence of actor nodes with a minimum spectrum access. In [13, the channel selection problem is investigated for cognitive radio based smart grid communications in the distribution section.

Furthermore, several recent studies have emphasized the need to address the optimal selection of transmission parameters in cognitive radio decision engine (CRDE) systems for non-smart grid applications using evolution algorithms. In [21, a multi-objective genetic-algorithm (GA) is presented to select the optimal communication transmission parameters. However, the result indicates that the GA has a slow convergence for the given communication modes. In [22, a population adaptation technique is used for the GA to decrease the time required to reach the final decision. The authors attempted to improve the work presented in 21] by taking the advantages of feedback learning from previous cognition cycles to speed up the system convergence. The algorithm starts at a high initial fitness that leads to a faster convergence than a standard GA. However, at high seeding values, the resulting fitness is lower than the fitness obtained with the standard GA. In 23, 24, a two-dimensional structure for chromosome's implementation of the GA was used to optimize the parameters of a CR engine. The results indicate that the non-dominated sorting GA has a faster convergence than the conventional GA. In [25, a mutated ant colony optimization (MACO)-based CR engine is proposed to find optimal transmission parameters. The results indicate that the fitness scores obtained by the MACO engine in the given communication scenarios are larger than those obtained by the conventional ant colony (AC) and GA engines. A cognitive radio adaptation method, which uses particle swarm optimization (PSO) as the decision method was proposed in [26. In this system, a discrete PSO was used to optimize parameters given a set of objectives for cognitive radios. In [27, a hybrid architecture of a cognitive decision engine based on the PSO algorithm and case study is proposed.
The case study can reduce the response delay of the cognitive radio engine and provides a radio with the ability to learn from its past running experiences. The result indicates that the hybrid PSO can achieve a better convergence than the original PSO and GA. However, this method requires a large storage for the efficient and considerably high performance.

These mentioned works generally refer to the CR engine optimization methods to optimize the transmission parameters based on the surrounding wireless communication environment. However, the required computation time to obtain optimal transmission parameters has been observed to be unrealistic for smart grid applications 28]. Furthermore, some studies have a trade-off between time convergence and scores of fitness function. Therefore, fast and accurate convergence optimization methods for optimal transmission parameters are required to achieve optimal results within a short period of time to support active communications.

\subsection{Study Contributions}

This paper attempts to fill the aforementioned gaps in the previous CR-related work. The contribution of this paper as compared to previous approaches can be summarized as follows.

First, a multi-objective differential evolution (MODE) optimization algorithm is proposed for the optimal selection of transmission parameters of a CR engine in a HAN for the home management application of a smart grid. The MODE provides fast convergence and a high score fitness function.

Second, four communication modes are adopted to utilize different environment conditions: power saving mode (minimum power), high throughput mode (maximum throughput), emergency communication mode (minimizing the bit error rate), and balanced mode (equal parameter priorities). The system adapts the transmission parameters dynamically according to the sensing wireless communication environments of the HAN. These modes are taken to address all environmental sensing statuses.

Third, the simulation results indicate that the proposed system can achieve higher fitness scores and faster convergence than other evolution algorithmbased CRDE such as; GA, PSO and MACO.

\section{Cognitive Radio Parameters}

The CR provides the ability to sense the surrounding wireless environment periodically and to adapt the transmission parameters appropriately according to the objectives for the optimal utilization of spectrum bands 29]. To achieve these goals, the CR needs a CRDE to provide efficient transmission parameters for the current environment, including transmission link, user demand, and system policies as shown in Fig. 1 . The CRDE must balance multiple objectives 30 . The environmental variables are responsible for enabling 


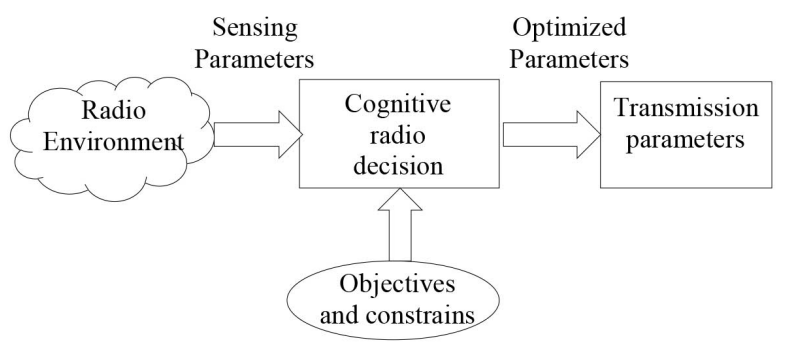

Figure 1. Cognitive radio system architecture.

the CR system to be alert to the surrounding environment to maximize the objectives of the system [31]. This information is periodically sensed by external sensors.

The three commonly used environmental parameters are the bit error rate (BER), signal-to-noise ratio (SNR) and noise power (N). The BER parameter represents the amount of erroneous bits in relation to the amount of bits being sent of a specific modulation type. The SNR represents the ratio of signal power to noise power 21. The transmission parameters are envisioned as system knobs that can be adapted based on the measured reading of environmental parameters and under the instruction of predefined objective functions.

In the context of optimization, these parameters can also be defined as decision variables that should be determined on the basis of the prescribed optimization procedures. The three parameters used to generate a fitness function are transmitting power $(P t)$, modulation type $(M T)$, and modulation index $(M I)$.

\section{OBjective FUnCtion}

The objective functions of a cognitive engine should be defined to guide the searching direction of optimization process for the optimal selection of the transmission parameters. In this work, three individual objective functions are combined to achieve a compromise among these objectives based on the predefined trade-off requirements. Table 1 presents the considered objectives of a CR system: minimum BER, maximum throughput and minimum transmitting power.

The fitness function of a minimum power consumption is defined as follows 21:

$$
f_{\text {min-power }}=1-\frac{P t}{P t_{\max }}
$$

where, $P t$ is the transmit power of the single carrier, and $P t_{\max }$ is the maximum available transmit power.

The fitness function of the maximum throughput is defined as follows [21]:

$$
f_{\text {min-throughput }}=\frac{\log _{10}(M I)}{\log _{10}\left(M I_{\max }\right)}
$$

where $M I$ is the modulation index of the given modulation types, and $M I_{\max }$ is the maximum modulation index used in the system. 21 .

The fitness function of minimum BER is given as

$$
f_{\text {min }-B E R}=1-\frac{\log _{10}(0.5)}{\log _{10}\left(P_{b e r}\right)}
$$

This fitness function is normalized to the worst possible BER value of (0.5) 32. Also, $P_{b e r}$ is the probability of a BER for a given modulation type. The probability of BER is calculated for M-ary phase-shift keying (PSK) and M-ary quadrature amplitude modulation (QAM) modulation types, as described by the following equations [21]:

For a binary PSK (BPSK) modulation type, the $P_{b e r}$ is

$$
P_{\text {ber_BPSK }}=Q\left(\sqrt{\frac{P t}{N}}\right), \quad M I=2
$$

For M-ary PSK modulation signal, the $P_{b e r}$ is

$$
\begin{aligned}
& P_{\text {ber_MPSK }}=\frac{2}{\log _{2}(M I)} Q \\
& \left(\sqrt{2 \cdot \log _{2}(M I) \cdot \frac{P t}{N} \cdot \sin \frac{\pi}{M I}}\right), \quad M I>2
\end{aligned}
$$

For M-ary QAM modulation signal, the $P_{b e r}$ is

$$
\begin{array}{r}
P_{b e r \_M Q A M}=\frac{4}{\log _{2}(M I)}\left(1-\frac{1}{\sqrt{M I}}\right) Q \\
\left(\sqrt{\frac{3 \cdot \log _{2}(M I)}{M I-1} \cdot \frac{P t}{N}}\right)
\end{array}
$$

where, $P t / N$ is the bit energy-to-noise power spectral density ratio.

These objective functions are obtained by dividing the variable score to its maximum possible value to attain a normalized range falling within $(0,1)$. This normalization can prevent the optimization trend from being attracted to the objectives of relatively large magnitudes. Each of the three objective functions is formulated to be a maximization problem by transforming any minimization objective $(f)$ to an equivalent $(1-f)$ maximization objective. The three single objective functions may interfere with each other. For instance, using a higher modulation index for a specific modulation scheme increases the throughput of the CR system, but it consequently increases the BER. Increasing the transmit power also reduces the BER thus improving the objective function of minimizing BER. However, this increment of the transmit power increases the power consumption, and thus reduces the objective function of minimizing the power consumption. Therefore, these conflicting objective functions need to be solved via the multi-objective optimization method. 


\begin{tabular}{llc}
\hline Objective name & Description & Related parameters \\
\hline Minimize power consumption & To decrease the amount of power consumption & $P t$ \\
Maximize throughput & To increase the data throughput & $M I$ \\
Minimize the bit-error rate & $\begin{array}{l}\text { To improve the overall BER of the transmission } \\
\text { environment }\end{array}$ & $P t, M I$ \\
\hline
\end{tabular}

TABLE 1. Cognitive radio objectives and related parameters.

\section{Multi-OBjective optimization}

Multi-objective optimization problems involve multiple conflicting objectives to be simultaneously optimized. Given the conflict objectives, a single solution that is simultaneously optimal with respect to all objectives does not necessarily exist [33]. A solution may be optimal for one objective, but sub-optimal or even poor for another. Therefore, a set of satisfied trade-off solutions known as Pareto optimal solutions is commonly used 34 . For such solutions, no improvement is possible for any objective without sacrificing at least one of the other objective functions. Thus, the main aim of the multi-objective optimization is to find the Pareto optimal solutions rather than the optimal solutions of independent objectives [35].

One of the issues that arise when solving multiobjective optimization problems is how to assign a single numerical single value for the overall multiobjective function in terms of its dependent objectives and corresponding variables. To realize this aim, preferences for individual objectives are first identified and numerically expressed so that the multiple objective functions can be placed in a single scalar multiobjective function 36 . The aggregation method has been used to combine multiple objective functions into one overall utility function. In this method, the optimization preferences are expressed by the weighting coefficients assigned to individual objective functions. Therefore, the aggregation method enables the combination of single-objective functions into a single multiple-objective function 37. The multi-objective function can be defined for the weighted sum of $\mathrm{m}$ objectives as 36 .

$$
f(x)=\sum_{i=1}^{m} w_{i} f_{i}(x)
$$

subject to $\sum_{i=1}^{m} w_{i}=1$ and $w_{i} \geq 0$ where, $m$ is the number of possible individual objective functions. For the current optimization problem (i.e., $m=3$ ), we can rewrite (7) as follows:

$$
\begin{array}{r}
f_{\text {multiple }}=w_{1} \cdot\left(f_{\text {min-power }}\right)+w_{2} \cdot\left(f_{\text {max-throughput }}\right)+ \\
+w_{3} \cdot\left(f_{\min -B E R}\right)
\end{array}
$$

where the weight vector $w_{i}$, determined by the three distinct CR transmission modes or scenarios, is defined by assigning a higher weight to a specific objective and lower weights to the others. In addition, another mode is introduced through a fair distribution of weights among the three objectives. Different modes for the weighing coefficients are defined by assigning a different weight to the fitness function of each objective. The aggregation (weighted sum) method is considered a flexible mechanism to steer the optimization process towards the highest priority objective of a higher weight. The resulting four modes (weight vectors) are identified in Table 2 for the comparison of results, as obtained in [25]. Each multi-objective fitness function is obtained by plugging in the corresponding weighing vector into Equation (8).

\section{Differential evolution}

Evolutionary multi-objective optimization (EMO) algorithms are powerful tools to solve multi-objective optimization and decision problems [38, and an EMO-based CRDE can support awareness-processing, decision-making, and learning elements of a cognitive functionality 29 . The differential evolution (DE) is a stochastic, population-based optimization algorithm developed by Storn and Price [39. The key difference between the DE and other evolutionary algorithms $(\mathrm{GA} / \mathrm{PSO})$ is in the mechanism for generating new solutions. Different from GA/PSO, the DE generates a new solution by combining several solutions with the candidate solution. The population in the DE evolves through repeated cycles of mutation, crossover, and selection, unlike the ones used in the GA 32 . The classical DE has four main stages: initialization, mutation, crossover, and selection [36]. Furthermore, three control parameters exist: $F, C r$, and $N P . F$ is the scaling factor that typically controls the differential mutation process between (0 and 1$)$. $\mathrm{Cr}$ is the crossover rate, which involves the probability that a trial vector is selected. $N P$ is the current population size, i.e., the number of competing solutions for any given generation $g$.

\subsection{INITIALIZATION}

The initial population should generate random individuals within the entire search space. The search space is prescribed by lower and upper bounds for each parameter of the optimization problem 40 .

The $i^{t h}$ parameter $(i=1,2, \ldots, D$, where $D$ is the total number of decision variables) of the $j^{\text {th }}$ individual vector $(j=1,2, \ldots, N P)$ at $g=1$ is initialized as follows [40]:

$$
x_{j, i}^{1}=x_{j, \min }+\operatorname{rand}_{j}(0,1)\left(x_{j, \max }-x_{j, \min }\right)
$$




\begin{tabular}{lcc}
\hline Transmission mode & Weighting vector $\left[w_{1}, w_{2}, w_{3}\right]$ & Mode \\
\hline $\begin{array}{l}\text { Power saving mode } \\
\text { (Minimum transmit power) }\end{array}$ & {$[0.8,0.05,0.15]$} & PSM \\
$\begin{array}{l}\text { High throughput mode } \\
\text { (Maximize throughput) }\end{array}$ & {$[0.15,0.8,0.05]$} & HTM \\
$\begin{array}{l}\text { Emergency communication mode } \\
\text { (Minimize bit-error rate) }\end{array}$ & {$[0.05,0.15,0.8]$} & ECM \\
$\begin{array}{l}\text { Balance mode } \\
\text { (Equal priority) }\end{array}$ & {$[1 / 3,1 / 3,1 / 3]$} & BLM \\
\hline
\end{tabular}

TABLE 2. Definition of CR transmission modes and weights [21, 22, 25, 27].

where, $\operatorname{rand}_{j}(0,1)$ is a random number within $(0,1)$ interval, which is multiplied by the interval length, $\left(x_{j, \max }-x_{j, \min }\right)$ to ensure a distributed sampling of the parameter's domain interval $\left[x_{j, \max }-x_{j, \text { min }}\right]$. Different approaches can be used to generate the initial population although random uniformity is the most common [41].

\subsection{Mutation}

Once the initialization is completed, the DE mutates and recombines the population to produce a population of NP mutant vectors. The differential mutation adds a scaled and randomly sampled vector difference to a third vector. Equation 10 shows how to combine three different randomly chosen vectors to create a mutant vector [36]:

$$
v_{i, g}^{1}=x_{r_{0}^{i}, g}+F \cdot\left(x_{r_{1}^{i}, g}-x_{r_{2}^{i}, g}\right)
$$

where $x_{r_{0}^{i}, g}, x_{r_{1}^{i}, g}$ and $x_{r_{2}^{i}, g}$ vectors are sampled randomly, selected from the current population rang and $r_{0}, r_{1}$, and $r_{2}$ are mutually exclusive integers randomly generated in the range $[1, N P]$, such that $r_{0} \neq r_{1} \neq r_{2} \neq i$. The mutation scale factor $F$ usually takes values within the range $[0,1]$ [42].

\subsection{Crossover}

The crossover enhances the potential diversity of a population. In a crossover, the trial vectors are produced according to [41.

$$
u_{j, i}^{g}= \begin{cases}v_{j, i}^{g} & \text { if }\left(\operatorname{rand}_{j}(0,1)\right) \leq C_{r} \text { or } j=j_{\text {rand }} \\ x_{j, i}^{g} & \text { otherwise }\end{cases}
$$

where $C_{r}$ is the crossover rate, which is a user-specified constant within the range, $[0,1]$, that controls the diversity of the population and enables the algorithm to escape from the local optima [40].

\subsection{Selection}

In the selection process, if the trial vector has an objective function value greater than or equal to the corresponding target vector, the target vector will be replaced by the trial vector and the last represents as a part of the population for the next generation. Otherwise, the target vector will remain in the population for the next generation. The selection operation can be expressed as follows [42]:

$$
x_{i}^{g+1}= \begin{cases}u_{i}^{g} & \text { if } f\left(u_{i}^{g}\right) \geq f\left(x_{i}^{g}\right) \\ x_{i}^{g} & \text { otherwise }\end{cases}
$$

Once the new population is constructed, the reproduction process (mutation, recombination, and selection) is repeated until the optimum solution is located, or a pre-specified termination criterion is satisfied, e.g., the number of generations reaches a pre-set maximum, gmax [36]. Fig. 2 shows the flowchart of the differential evolution.

\section{Proposed Mode-BASEd CRDE OF WIRELESS HAN}

A scenario considered, in which the CRDE functionality is deployed inside the smart meter, which is connected to the utility load management centre and to the HAN base station, to achieve optimal management for the customer's appliances is shown in Fig. 3 . The cognitive radio is proposed to select the optimal transmission parameters for the given communication modes within the HAN of the home management system.

Fig. 4 shows the proposed MODE-base CR system architecture. The CR system extracts the data of the sensing wireless channel conditions via its environmental parameters and passes these data to a multi-objective DE. The system weight generation of the CR transmission is used to assign a distinct weighting coefficient for given transmission modes. These different weighting coefficients are used to vary the priority level among radio objectives. The largest weighting coefficient is allocated for the objective of the highest priority as shown in table 2. The other benefit of these weighting coefficients is the conversion of the multi-objective optimization problem into a single objective optimization problem using the aggregation method. The sensed environmental parameters and the weighting coefficients are assigned to three transmission mode objectives to construct the multiobjective function. The DE optimization engine is 


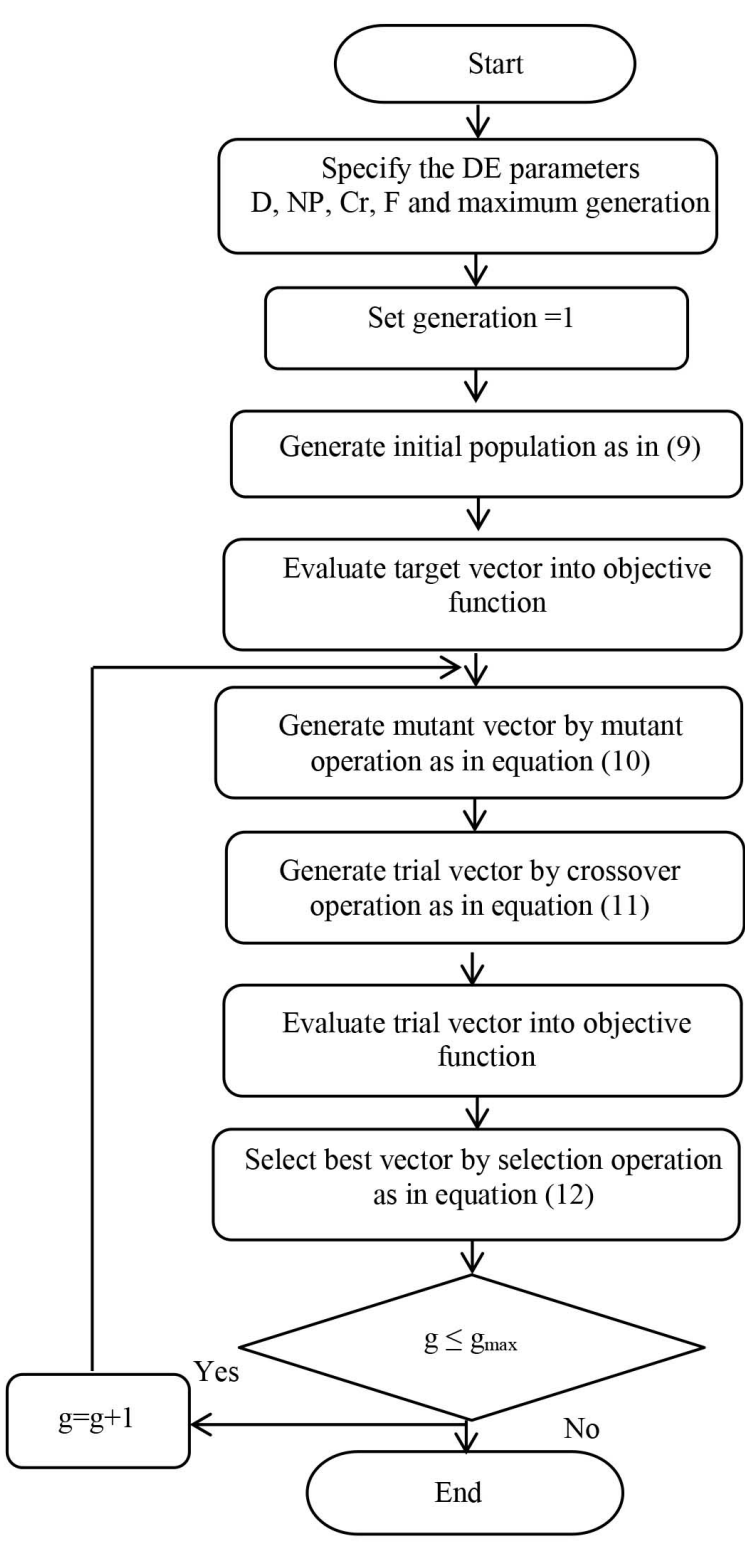

FIGURE 2. Flowchart of differential evolution.

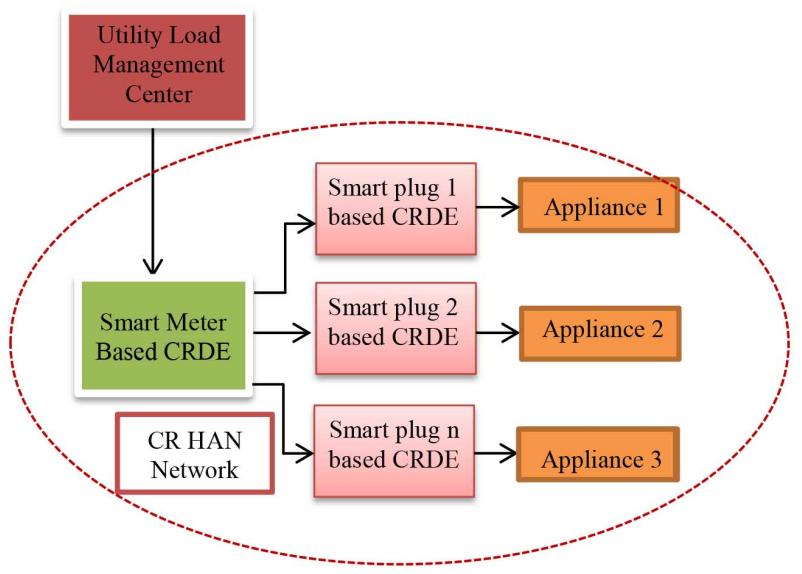

Figure 3. Proposed CRDE for HAN of smart grid.

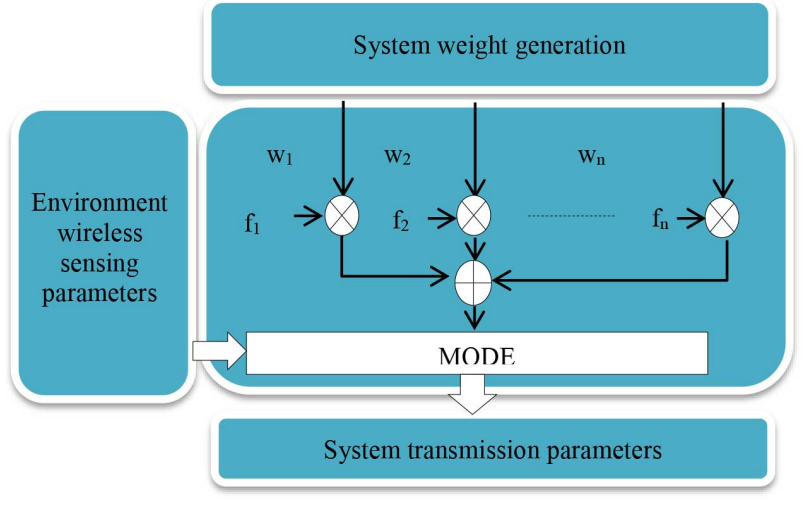

Figure 4. Proposed MODE-base CR system architecture.

used to find an optimal set of transmission parameters. Based on the selected communication mode, the communication in the home load management system of HAN is established.

\subsection{Simulation Results}

The proposed MODE cognitive decision engine is simulated using the MATLAB software package. The DE parameters used in this work are based on the recommended values in [43, $(F=0.85, \mathrm{Cr}=0.6$ and $N P=10 D)$. For the sake of simplicity, only unlicensed bands are considered in the HAN of the home management system. Therefore, the transmitted power ranges from $0.1 \mathrm{~mW}$ to $2.56 \mathrm{~mW}$ in increments of $0.0256 \mathrm{~mW}$. This range of the transmit power is close to the maximum transmit power level allowed in the unlicensed national information infrastructure (UN-IIB) band 21. However, the proposed CRDE and its results can be extended to other spectrum bands (e.g., licensed or hydride bands).

The modulation index (MI) is selected to be any of the nine possible values 2-512. Practically, the PSK is commonly used for low modulation indices (i.e. when MI equals to or is less than 8), whereas the QAM is used for high modulation indices. Thus, the proposed modulation schemes used in this work are 2-PSK (or BPSK), 4-8 PSK, 16-512 QAM. The proposed choices of modulation type (i.e., types of plus indices) offer the modulation diversity needed for the $\mathrm{CR}$ adaption requirement.

The performance of the MODE-based CRDE system is simulated under the four transmission modes and presented in Table 3. The convergence performance of the power saving mode (PSM) is shown in Fig. 5. The simulation graph presents the convergence progresses of the mean (average) of over 10 simulation runs to provide a time-invariant average and maximum (best) fitness with respect to the number of generations. The maximum achievable fitness under this mode is 0.964 , and it is captured after three generations as indicated in Table 3. The Pareto-front solution achieved for transmission parameters' for PSM returns a transmit power of $0.1 \mathrm{~mW}$ and a modulation 


\begin{tabular}{cccccccc}
\hline $\begin{array}{c}\text { Transmission } \\
\text { mode }\end{array}$ & $\begin{array}{c}\text { Fitness } \\
\text { scores }\end{array}$ & $\begin{array}{c}\text { No. of } \\
\text { generation } \\
\text { (Max. fitness) }\end{array}$ & $\begin{array}{c}\text { No. of } \\
\text { generation } \\
\text { (Mean. fitness) }\end{array}$ & $\begin{array}{c}\text { Time } \\
\text { per one } \\
\text { generation } \\
(\mathrm{ms})\end{array}$ & MT & MI & Pt/mw \\
\hline PSM & 0.9648 & 3 & 8 & 1.51 & QAM & 512 & 0.1 \\
HTM & 0.9880 & 2 & 7 & 1.54 & QAM & 512 & 1.253 \\
ECM & 0.9629 & 3 & 9 & 1.57 & PSK & 4 & 2.56 \\
BLM & 0.9596 & 2 & 5 & 1.63 & QAM & 512 & 0.125 \\
\hline
\end{tabular}

TABLE 3. Simulation results of DE-based CR system.

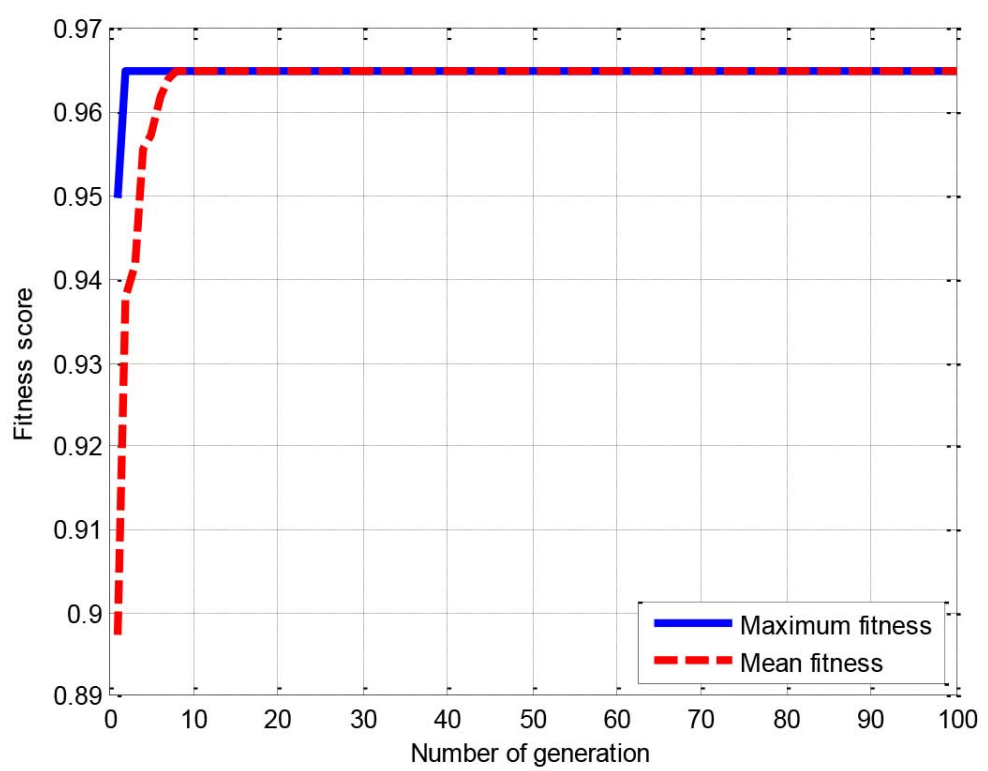

Figure 5. Power saving mode (PSM) convergence.

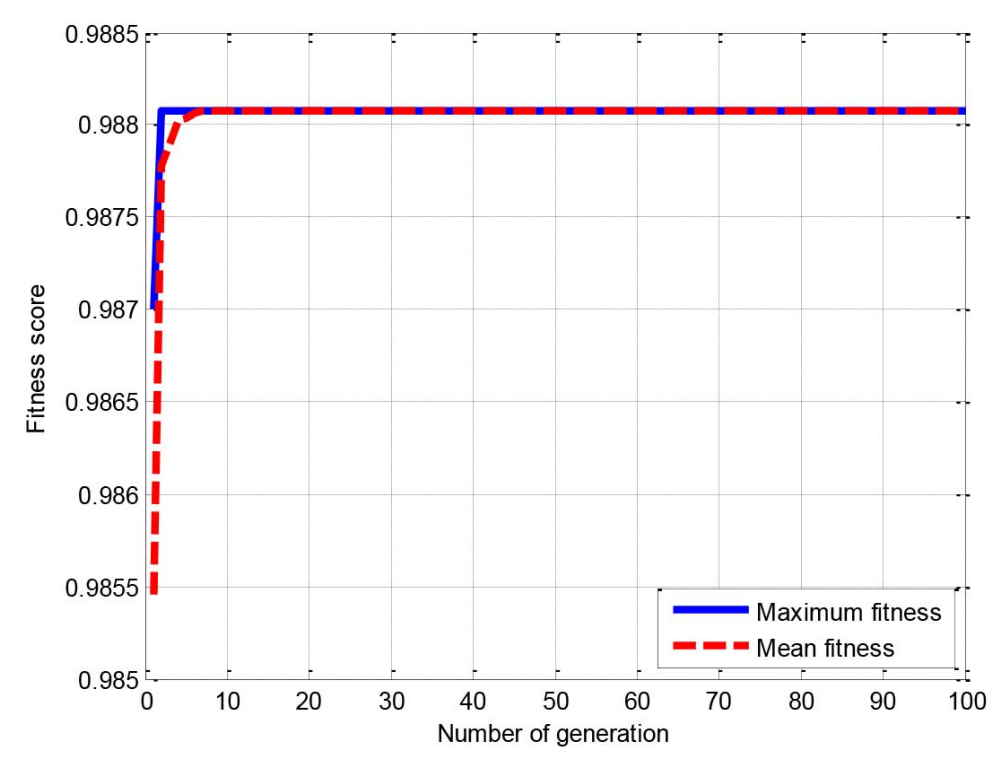

FIGURE 6. High throughput mode (HTM) convergence. 


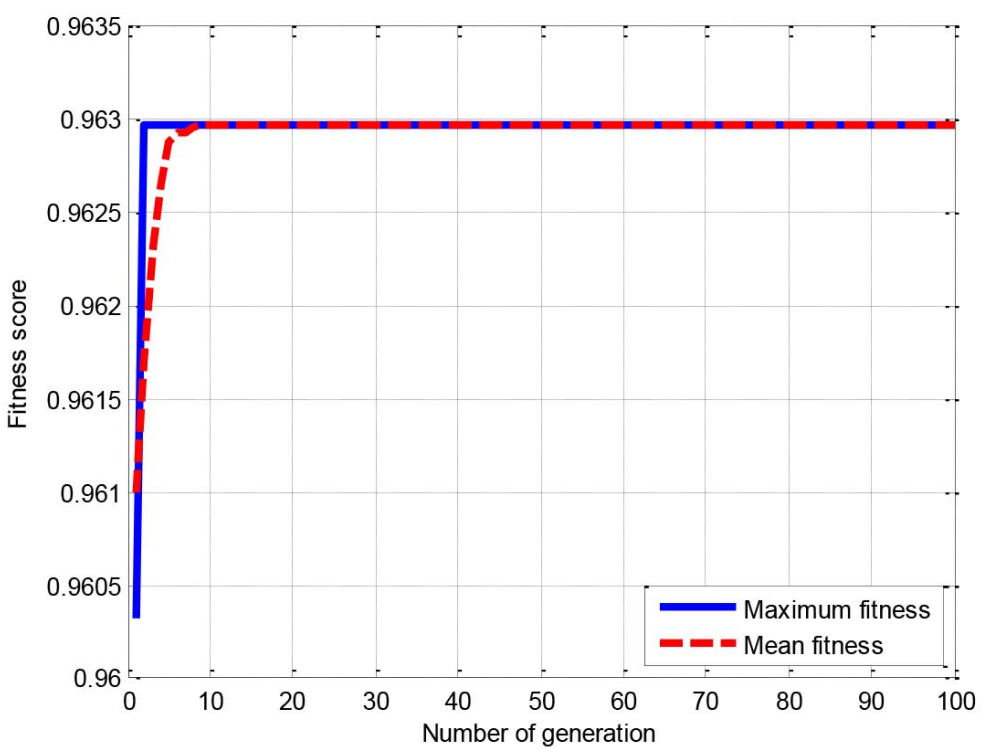

FIgURE 7. Emergency communication mode (ECM) convergence.

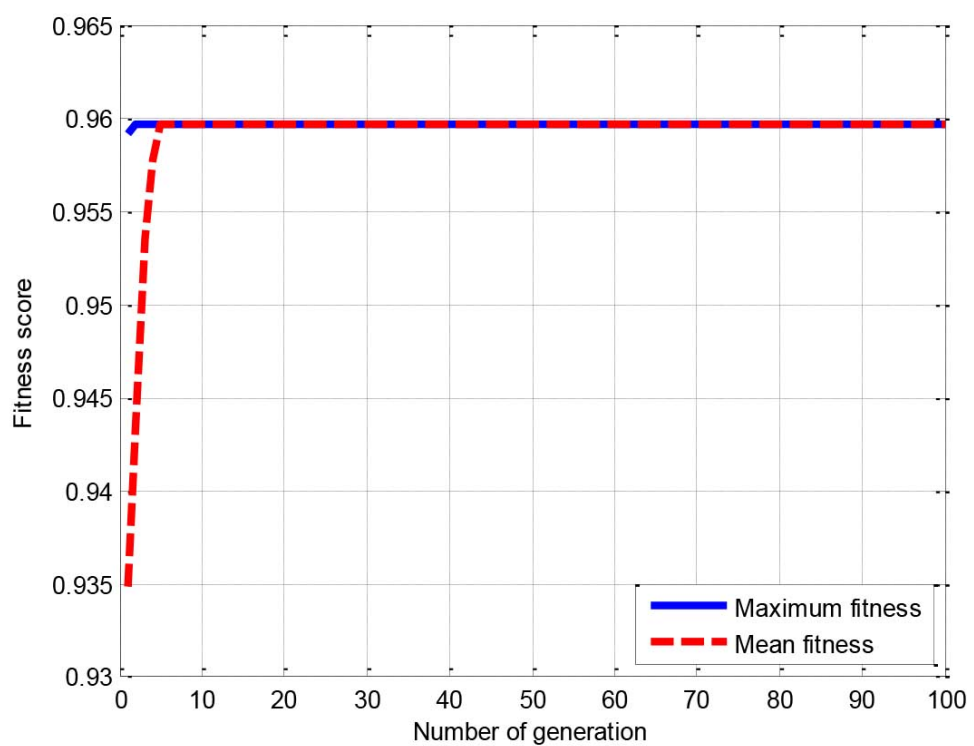

FigURE 8. Balance communication mode (BLM) convergence.

scheme of 512-QAM. The transmit power controlled by the MODE cognitive engine is much lower (to save the power), while the modulation scheme is relatively high (which means a high BER). When a large amount of data must be sent or relayed while maintaining a high throughput, the weighting coefficients of the high throughput mode (HTM) function are distributed to prioritize the maximizing throughput. The results are obtained after a fast convergence of only two generations, scoring a fitness value of 0.988 (see Fig. 6). For this communication mode, the obtained Pareto-front solution are is a transmit power of $1.253 \mathrm{~mW}$ and a modulation scheme of 512-QAM. Under this mode, therefore, the modulation scheme is set to 512-QAM by the MODE cognitive engine, which maximizes the throughput of the systems at the cost of a large transmit power and a high BER. Under the emergency communication mode (ECM), additional emphasis is placed on the objective of minimizing the BER to realize a scenario of a reliable transmission and reception. Fig. 7 illustrates the results obtained by running the MODE-based CRDE system for a given set of environmental parameters. The maximum achievable fitness is 0.962 , and it is obtained within the three generations. As shown in Table 3 , the Pareto-front results returns a transmit power of $2.56 \mathrm{~mW}$ and a modulation scheme of 4-PSK for the ECM communication mode. The modulation scheme is extremely low, controlled by the MODE cognitive engine at the cost of a larger transmit power. For the balanced priority mode (BLM), the three single objective functions constructed are all given equal weights. This scenario may be utilized by the MODE-based CRDE system in the case no particular operational plan exists or when 
the system requires no major attraction in favour of a specific objective compared with other objectives. The convergence performance of the system is shown in Fig. 8 Figure 8 demonstrates the optimal solution obtained after only two generations, scoring a maximum achievable fitness of 0.959 . Under this communication mode, the Pareto-front solution provides a transmit power of $0.125 \mathrm{~mW}$ and a modulation scheme of 512-QAM. The transmit power is relatively small with a high modulation scheme. Another important characteristic of the proposed MODE-based CRDE communication system is the time per generation. The cognitive system is stopped after it reaches the generation that gives the highest possible fitness scores. For all considered communication modes in Table 3 , the system reaches the highest scoring fitness after only two to three generations. The average times per one generation are $1.51,1.54,1.57$, and $1.63 \mathrm{~ms}$ for PSM, HTM, ECM, and BLM, respectively. Therefore, the total required times for PSM, HTM, ECM, and BLM are 4.53, 3.08, 4.71, and $3.26 \mathrm{~ms}$, respectively, to complete the computation within two DE generations.

\subsection{Performance COMPARISON}

To highlight the difference between the proposed MODE-based CRDE and previously published systems, the proposed MODE-based CRDE is compared with the GA-based CR systems used in 21, the MACO-based CR in 25] and the PSO based CR in 26. The comparison is based on the maximum fitness scores for the given communication modes and the number of generations to reach the maximum fitness scores. For the PSM, the GA-based CR had a maximum fitness of 0.93 that was reached within approximately 400 generations. For the MACO-based system, the provided maximum fitness was 0.9482 , which was reached within approximately 470 generations. Meanwhile, the maximum score was 0.944 reached within 300 generations using the PSO-based system. On the contrary, the proposed MODE-based CRDE offers a maximum fitness of 0.964 that is reached within three generations. In the HTM communication mode, the fitness score of the GA-based was 0.938, which was obtained within around 200 generations. The fitness function score of the MACO-based system was approximately 0.9422 and was obtained within 470 generations. For the PSO-based system the fitness score was about 0.944 obtained within 150 generations. In the MODE-based approach, a fitness score of 0.988 was reached within only two generations. The comparison of the convergence performance of each CR system is presented in Table 4 for the given communication modes. The proposed MODE-based CRDE had larger fitness scores for all of the communication modes compared with the other CRDE systems as shown in Table 4 Furthermore, the MODE-based CRDE had a faster convergence; the maximum fitness with optimal parameters for different communication modes was reached within only two to three generations. The
MODE provides fast and accurate convergence, which is a critical issue for the cognitive radio to adapt the transmission parameters with respect to the changes in the wireless environment.

\subsection{Discussion}

The results of the proposed MODE-based CRDE indicate that the convergence towards the optimal communication transmission parameters is essentially fast and accurate. The results of the proposed MODEbased CRDE refer the convergence toward the optimal transmission parameters is essentially fast and accurate. For various communication modes, the proposed system reaches the optimal parameters within only two to -three generations of the DE to support the active and rapid communication response. The time required to reach the final higher scored fitness function is another key parameter of the proposed MODE-based CRDE system. For the given four-communication modes, the average time per one generation is about $1.5 \mathrm{~ms}$. Furthermore, the fitness scores of the given modes are higher than $95 \%$, thus supporting the accuracy requirement of active communication systems. The fast and accurate convergences are the most important key parameters of the proposed MODE-based CRDE system. For Regarding the obtained Paretofront solutions for given communication modes, are they tend to match the target objective for each mode while scarify sacrificing the other objectives.

According to results, the proposed MODE-based CRDE performs an optimal management for the originally scarce spectrum resources of the in-home wireless communication of smart grids. This system helps customers manage and schedule the available appliances for the given optimal signals of a home managementbased utility pricing scheme. Moreover, the proposed CR communication system maintains the essential use of the wireless spectrum environment of the HAN.

\section{Conclusion}

A MODE-based CRDE system is proposed to adapt the transmission parameters according to the sensing parameters of a wireless environment in the HAN of a smart grid. Multi-conflicting objectives have been aggregated into a one overall multi-objective function using variable weighting coefficients that can be adjusted to define distinct transmission scenarios.

The selection among these transmission scenarios is assumed to be based on user preferences or possibly on environmental conditions. These distinct transmission modes are PSM, HTM, ECM, and BLM. The proposed DE decision engine is developed to enable the optimization of the transmission parameters for a given set of sensed environmental parameters that can satisfy the requirements of the predefined transmission mode.

The performance of the proposed MODE-based CRDE system is evaluated and verified under the proposed transmission modes. The proposed MODE 


\begin{tabular}{ccccccccc}
\hline \multirow{2}{*}{$\begin{array}{c}\text { Proposed } \\
\text { modes }\end{array}$} & \multicolumn{2}{c}{ GA-based CR [21] } & \multicolumn{2}{c}{ MACO-based CR [25] } & \multicolumn{2}{c}{ PSO-based CR [26] } & \multicolumn{2}{c}{ Proposed } \\
& Fitness & No. of & Fitness & No. of & Fitness & No. of & Fitness & No. of \\
sen. & score & gen. & score & gen. & gen. \\
\hline PSM & 0.930 & 400 & 0.9482 & 470 & 0.9444 & 300 & 0.9648 & 3 \\
HTM & 0.938 & 200 & 0.9422 & 470 & 0.9444 & 150 & 0.9880 & 2 \\
ECM & 0.800 & 400 & 0.8523 & 470 & 0.7776 & 300 & 0.9629 & 3 \\
BLM & - & - & 0.8460 & 470 & 0.8765 & 150 & 0.9596 & 2 \\
\hline
\end{tabular}

TABLE 4. Comparison of convergence performance.

decision engine can converge to the optimal of transmission solutions within only two to three evolutionary generations, with fitness scores greater than $95 \%$ for all of the communication modes. Furthermore, the total time required to reach the higher scored fitness for given communication modes is approximately $4.5 \mathrm{~ms}$. The results exhibit the superiority of the proposed MODE-based CRDE systems in terms of accuracy and convergence as compared to previous works presented in literature. The convergence speed and accuracy of the fitness function make the proposed MODE decision engine feasible for a CR application of a home management system of a smart grid [28]. In the upcoming work, a multi-criteria decision making (MCDM) method will be used to sort all possible optimal communication parameters from the best to the worst, based on predefined criteria.

\section{ACKNOWLEDGEMENTS}

The authors would like to thank Mustansiriyah University (www.uomustansiriyah.edu.iq) Baghdad-Iraq for its support with the present work.

\section{REFERENCES}

[1] H. Tarish Haider, O. Hang See, W. Elmenreich. A review of residential demand response of smart grid. Renewable and Sustainable Energy Reviews 59:166-178, 2016. DOI:10.1016/j.rser.2016.01.016

[2] R. Lu, S. Hong. Incentive-based demand response for smart grid with reinforcement learning and deep neural network. Applied Energy 236:937-949, 2019. DOI:10.1016/j.apenergy.2018.12.061

[3] S. Hong, M. Yu, X. Huang. A real-time demand response algorithm for heterogeneous devices in buildings and homes. Energy 80:123-132, 2014. DOI:10.1016/j.energy.2014.11.053

[4] H. Tarish Haider, O. Hang See, W. Elmenreich. Optimal residential load scheduling based on time varying pricing scheme. In 13th IEEE Student Conference on Research 85 Development 2015 (SCOReD), pp. 210-214. Kuala Lumpur, 2015. DOI:10.1109/SCORED.2015.7449326.

[5] M. E. Bayrakdar, A. Çalhan. Artificial bee colonybased spectrum handoff algorithm in wireless cognitive radio networks. International Journal of Communication Systems 31:1-16, 2018. DOI:10.1002/dac.3495.
[6] M. Barbeau, G. Cervera, J. Garcia-Alfaro, E. Kranakis. Channel selection using a multiple radio model. Journal of Network and Computer Applications 64:113-123, 2016. DOI:10.1016/j.jnca.2016.01.021.

[7] M. E. Bayrakdar, A. Çalhan. Non-preemptive queueing model of spectrum handoff scheme based on prioritized data traffic in cognitive wireless networks. ETRI Journal 39:558-569, 2017. DOI:10.4218/etrij.17.0116.0850

[8] S. Alam, M. F. Sohail, S. A. Ghauri, et al. Cognitive radio based Smart Grid Communication Network. Renewable and Sustainable Energy Reviews 72:535-548, 2017. DOI:10.1016/j.rser.2017.01.08.

[9] U. Khan, N. Dilshad, M. H. Rehmani, T. Umer. Fairness in cognitive radio networks: Models, measurement methods, applications, and future research directions. Journal of Network and Computer Applications 73:12-26, 2016. DOI:10.1016/j.jnca.2016.07.008

[10] O. Bicen, V. Cagri Gungor, O. Akan. Delay-sensitive and multimedia communication in cognitive radio sensor networks. Ad Hoc Networks 10:816-830, 2012. DOI:10.1016/j.adhoc.2011.01.021

[11] J. Palicot, C. Moy, B. Résimont, R. Bonnefoi. Application of hierarchical and distributed cognitive architecture management for the smart grid. Ad Hoc Networks 41:86-98, 2016. DOI:10.1016/j.adhoc.2015.12.002

[12] M. Faheem, S. Shah, R. Butt, et al. Smart grid communication and information technologies in the perspective of Industry 4.0: Opportunities and challenges. Computer Science Review 30:1 - 30, 2018. DOI:10.1016/j.cosrev.2018.08.001.

[13] S. Althunibat, Q. Wang, F. Granelli. Flexible channel selection mechanism for cognitive radio based last mile smart grid communications. Ad Hoc Networks 41:47 - 56, 2016. DOI:10.1016/j.adhoc.2015.10.008.

[14] O. Ergul, O. Bicen, O. Akan. Opportunistic reliability for cognitive radio sensor actor networks in smart grid. Ad Hoc Networks 41:1-10, 2015. DOI:10.1016/j.adhoc.2015.10.003

[15] U. Premarathne, I. Khalil, M. Atiquzzaman. Trust based reliable transmission strategies for smart home energy management in cognitive radio based smart grid. Ad Hoc Networks 41:15-29, 2016. DOI:10.1016/j.adhoc.2015.12.004 
[16] A. Abuadbba, I. Khalil, A. Ibaida, M. Atiquzzaman. Resilient to shared spectrum noise scheme for protecting cognitive radio smart grid readings - $\mathrm{BCH}$ based steganographic approach. Ad Hoc Networks 41:30-46, 2016. DOI:10.1016/j.adhoc.2015.11.002

[17] U. Premarathne, I. Khalil, M. Atiquzzaman. Secure and reliable surveillance over cognitive radio sensor networks in smart grid. Pervasive and Mobile Computing 22:3-15, 2015. DOI:10.1016/j.pmcj.2015.05.001.

[18] H. Wang, Y. Qian, H. Sharif. Multimedia communications over cognitive radio networks for smart grid applications. IEEE Wireless Communications 20:125-132, 2013. DOI:10.1109/MWC.2013.6590059.

[19] A. Saghiri, M. Meybodi. An approach for designing cognitive engines in cognitive peer-to-peer networks. Journal of Network and Computer Applications 70:17-40, 2016. DOI:10.1016/j.jnca.2016.05.012

[20] W. Khan, M. Zeeshan. QoS-based dynamic channel selection algorithm for cognitive radio based smart grid communication network. Ad Hoc Networks 87:61-75, 2019. DOI:10.1016/j.adhoc.2018.11.007.

[21] T. R. Newman, B. A. Barker, A. M. Wyglinski, et al. Cognitive engine implementation for wireless multicarrier transceivers. Wireless Communications and Mobile Computing 7:1129-1142, 2007. DOI:10.1002/wcm.486

[22] T. R. Newman, R. Rajbanshi, A. M. Wyglinski, et al. Population adaptation for genetic algorithm-based cognitive radios. Mobile Networks and Applications 13:442-451, 2008. DOI:10.1007/s11036-008-0079-8

[23] C. Huynh, W. Lee. Multicarrier cognitive radio system configuration based on interference analysis by two dimensional genetic algorithm. In Advanced Technologies for Communications (ATC), 2011 International Conference, pp. 85-88. Da Nang, Vietnam, 2011. DOI:10.1109/ATC.2011.6027441.

[24] C. K. Huynh, W. C. Lee. Two-dimensional Genetic algorithm for OFDM-based cognitive radio systems. In 2011 IEEE 3rd International Conference on Communication Software and Networks (ICCSN), pp. 100-105. 2011. DOI:10.1109/ICCSN.2011.6013553

[25] N. Zhao, S. Li, Z. Wu. Cognitive radio engine design based on ant colony optimization. Wireless Personal Communications 65:15-24, 2012. DOI:10.1007/s11277-011-0225-7.

[26] Z. Zhao, S. Xu, S. Zheng, J. Shang. Cognitive radio adaptation using particle swarm optimization. Wireless Communications and Mobile Computing 9:875-881, 2009. DOI:10.1002/wcm.633

[27] X. Tan, H. Zhang, J. Hu. A hybrid architecture of cognitive decision engine based on particle swarm optimization algorithms and case database. Annals of Telecommunications - Annales des Télécommunications 69:593-605, 2014. DOI:10.1007/s12243-013-0417-0

[28] V. C. Gungor, D. Sahin, T. Kocak, et al. A survey on smart grid potential applications and communication requirements. IEEE Transactions on Industrial Informatics 9(1):28-42, 2013. DOI:10.1109/TII.2012.2218253
[29] E. Palacios-García, A. Chen, I. Santiago, et al. Stochastic model for lighting's electricity consumption in the residential sector. Impact of energy saving actions. Energy and Buildings 89:245-259, 2015. DOI:10.1016/j.enbuild.2014.12.028.

[30] W. Chen, T. Li, T. Yang. Intelligent control of cognitive radio parameter adaption: Using evolutionary multi-objective algorithm based on user preference. Ad Hoc Networks 26:3-16, 2015. DOI:10.1016/j.adhoc.2014.09.006

[31] E. Hossain, D. Niyato, D. In Kim. Evolution and future trends of research in cognitive radio: $\mathrm{A}$ contemporary survey. Wireless Communications and Mobile Computing 15:1530-1564, 2013. DOI:10.1002/wcm.2443

[32] P. M. Pradhan, G. Panda. Comparative performance analysis of evolutionary algorithm based parameter optimization in cognitive radio engine: A survey. Ad Hoc Networks 17:129-146, 2014. DOI:10.1016/j.adhoc.2014.01.010

[33] E.-G. Talbi. Metaheuristics: from design to implementation, vol. 74. John Wiley \& Sons, Hoboken, New Jersey, USA, 2009.

[34] G.-Q. Zeng, J. Chen, L.-M. Li, et al. An improved multi-objective population-based extremal optimization algorithm with polynomial mutation. Information Sciences 330:49-73, 2016. DOI:10.1016/j.ins.2015.10.010.

[35] C. A. C. Coello, D. A. Van Veldhuizen, G. B. Lamont. Evolutionary algorithms for solving multi-objective problems. Springer Science \& Business Media, New York, USA, 2002. DOI:10.1007/978-1-4757-5184-0

[36] K. V. Price, R. Storn, J. Lampinen. Differential Evolution: A Practical Approach to Global Optimization. Springer-Verlag Berlin Heidelberg, 2005. DOI:10.1007/3-540-31306-0

[37] R. Marler, J. Arora. The weighted sum method for multi-objective optimization: New insights. Structural and Multidisciplinary Optimization 41:853-862, 2010. DOI:10.1007/s00158-009-0460-7.

[38] M. A. Fotouhi Ghazvini, P. Faria, S. Ramos, et al. Incentive-based demand response programs designed by asset-light retail electricity providers for the day-ahead market. Energy 82:786-799, 2015. DOI:10.1016/j.energy.2015.01.090

[39] R. Storn, K. Price. Differential evolution - A simple and efficient heuristic for global optimization over continuous spaces. Journal of Global Optimization 11:341-359, 1997. DOI:10.1023/A:1008202821328.

[40] M. Ali, N. Awad, P. Suganthan. Multi-population differential evolution with balanced ensemble of mutation strategies for large-scale global optimization. Applied Soft Computing 33:304-327, 2015. DOI:10.1016/j.asoc.2015.04.019

[41] C. Roque, P. Martins. Differential evolution for optimization of functionally graded beams. Composite Structures 133:1191-1197, 2015. DOI:10.1016/j.compstruct.2015.08.041 
[42] A. K. Qin, V. L. Huang, P. N. Suganthan.

Differential evolution algorithm with strategy

adaptation for global numerical optimization. IEEE

Transactions on Evolutionary Computation 13:398-417, 2009. DOI:10.1109/TEVC.2008.927706.

[43] V. Feoktistov. Differential evolution - in search of solutions 5, 2006. DOI:10.1007/978-0-387-36896-2 